\title{
A generalized Stefan model accounting for system memory and non-locality
}

\author{
R. Garra ${ }^{1}$ \\ BCAM - Basque Center for Applied Mathematics, Alameda de Mazarredo 14, E-48009 \\ Bilbao, Basque Country, Spain \\ F. Falcini \\ ISMAR-CNR, Via Fosso del Cavaliere, 100, 00133 Rome, Italy \\ V.R. Voller \\ Department of Civil, Environmental, and Geo- Engineering, University of Minnesota, 500 \\ Pillsbury Drive SE, Minneapolis, MN 55455, USA \\ G. Pagnini \\ Ikerbasque - Basque Foundation for Science, Calle de María Díaz de Haro 3, E-48013 \\ Bilbao, Basque Country, Spain
}

\begin{abstract}
The Stefan problem, involving the tracking of an evolving phase-change front, is the prototypical example of a moving boundary problem. In basic onedimensional problems it is well known that the front advances as the square root of time. When memory or non-locality are introduced into the system however, this classic signal may be anomalous; replaced by a power-law advance with a time exponent that differs from $n=1 / 2$. Up to now memory treatments in Stefan problem models have only been able to reproduce sub-diffusive front movements with exponents $n<1 / 2$ and non-local treatments have only been able to reproduce super-diffusive behavior $n>1 / 2$. In the present paper, using a generalized Caputo fractional derivative operator, we introduce new memory and non-local treatment for Stefan problems. On considering a limit case Stefan
\end{abstract}

\footnotetext{
${ }^{1}$ Corresponding author.

E-mail address: roberto.garra@sbai.uniroma1.it (R. Garra)
}

Preprint submitted to Int. Commun. Heat Mass Transf

February 5, 2019 
problem, related to the melting problem, we are able to show that, this general treatment can not only produce arbitrary power-law in time predictions for the front movement but, in the case of memory treatments, can also produce non-power-law anomalous behaviors. Further, also in the context of the limit problem, we are able to establish an equivalence between non-locality and a space varying conductivity and memory and a time varying conductivity.

Keywords: Stefan problems, fractional moving boundary problems, melting processes, anomalous diffusion.

\section{Introduction}

A classical free boundary problem, generally referred as the Stefan problem [1], concerns the study of the transient diffusion-controlled melting/solidification (phase change) of a solid/liquid adjacent to a heated/cooled surface. In the one-

5 dimensional form of this problem, the time-dependent position of the liquid-solid interface $s(t)$, relative to the heated/cooled surface, advances as the square root of time, $s(t) \sim t^{n}, n=1 / 2$ [], which is the expected normal behavior for a diffusion process. Experimental observations of Stefan like and related phasechange problems, e.g., frost growth [2] and moisture infiltration [3, however, indicate that in some physical settings different time exponents, i.e., $n>1 / 2$ (super-diffusive) or $n<1 / 2$ (sub-diffusive), can manifest. These observations have motivated theoretical studies to investigate general Stefan models formulations that can produce anomalous behaviors [4, 5, 6, 17, 8, 9, 10. We also refer to the recent discussion paper [1] and the references therein about fractional Stefan problems.

The recent work in [12] provides an extensive review on the theory and applications of anomalous behavior in heat transfer system. One method to treat anomalous behavior is by the introduction of a memory into the system of interest, requiring that the current state of the system depends on a weighting (usually decaying) of previous states in time; this can be achieved in Stefan models by replacing the transient terms in the governing equations 
with a $0<\nu \leq 1$ order time-fractional derivative, representing a convolution in time [4, 5, 6, 7, 8, 9]. An alternative approach is to invoke a non-local behavior in which the operation of a process at a given space point in the system depends not just on the conditions at that point but also on a weighting of the current conditions throughout the whole domain; this can be achieved in Stefan models by replacing the temperature gradient in the heat flux definition with a $0<\nu \leq 1$ order space-fractional derivative representing a convolution in space [4, 7, 8, 9]. Voller [8, working with a limit case Stefan problem related to melting process, investigates how replacing the transient and gradient terms with Caputo fractional derivatives [13] of order $0<\nu<1$, representing, in turn, the effects of memory (time-fractional) and non-locality (space-fractional), produces predictions for the power-law advance of the phase front exhibiting a range of exponents i.e., $0 \leq n \leq 1$. Replacing the transient term with a time-fractional derivative (memory) results in a sub-diffusive power-law front advance $s=t^{n}$, $0 \leq n \leq 1 / 2$. In contrast, replacing the gradient term with a space-fractional derivative (non-local), produces a super-diffusive advance $s=t^{n}, 1 / 2 \leq n \leq 1$. We stress the point that predictions from a Stefan model with a fractional time derivative are restricted to sub-diffusive anomalous behavior while predictions from models with a fractional gradient term are restricted to super-diffusive anomalous behavior.

Outside of using fractional calculus models, however, anomalous behaviors for the phase front movement from a Stefan problem can also be obtained by using a space and/or time dependent thermal conductivity in a conventional

${ }_{45}$ (integer derivative) Fourier heat transport equation. For example, on using a power-law in space definition of the conductivity (e.g., a graded material), Falcini and Voller [10] derive a closed analytical solution of a one-dimensional Stefan problem in which the phase front movement recovers the full range of power-law in time exponents, $0 \leq n \leq 1$. In more recent work, Falcini et al. [4, working with a generalized Fourier heat transport model that combines both Caputo space and time fractional derivatives with a power-law in space dependent conductivity, explore the connections between memory, non-locality, and 
variable conductivity in setting the space-time scaling for diffusion controlled problems. While this work provides a theoretical underpinning for the anomalous behaviors associated with generalized Stefan models it also introduces some ambiguity related to matching a given model choice (memory, non-local, variable conductivity) to a given observation of the phase front movement; e.g., as shown in [4], with the appropriate choice of time derivative order or powerlaw spacial variation of conductivity, both memory and non-linear conductivity models can predict a power-law in time phase front movement $s=t^{n}$ with an identical sub-diffusive exponent $0 \leq n \leq 1 / 2$.

At this point, we should recognize that anomalous diffusion signals are not restricted to the form of a power-law in time but more correctly include any signals whose variance does not grow linearly in time. In this light, another interesting class of anomalous diffusion processes to consider is ultraslow scaled Brownian processes, where the variance grows logarithmically in time (see for example [14] and the references therein). Recovering such a behavior for the phase front movement, however, is out of the reach of the current memory, nonlocal and variable conductivity treatments that have been proposed for Stefan models.

The objective of this paper is to introduce a general, fractional based treatments for modeling memory and non-locality in a Stefan problem. The key step is to replace the standard Caputo fractional derivative of temperature with respect to time $t$ or space $x$, used in previous general Stefan model treatments, with an integro-differential evolution operator that is essentially the Caputo fractional derivative of temperature with respect to a general function of time $f(t)$ or space $f(x)$. With appropriate settings, using these treatments in the Stefan problem, even in the absence of a space or time varying conductivity, can not only predict phase front movements with arbitrary positive power-law 80 time exponents $0 \leq n \leq 1$ but can also predict front movements that exhibit non-power-law anomalous behaviors. Further, in the context of the limit melting Stefan problem introduced in Voller [8], we are able to mitigate some of the ambiguity in matching a suitable model treatment to given observations of 
front movement and temperature profile. In particular, we show, in this limit problem, (i) that non-local treatments are essentially equivalent to treatments that use a spatially varying conductivity (representing a graded material), (ii) that memory treatments are essentially equivalent to treatments that use a time varying conductivity (representing an aging effect), and (iii) a clear delineation of anomalous behaviors resulting from memory and non-local treatments.

\section{A one-phase, one-dimensional Stefan model}

Without too much loss of generality we will carry our arguments in this work by considering a one-phase, one-dimensional Stefan problem. This involves the melting of a solid in a one-dimensional domain $x \geq 0$. Initially the solid is at the unique phase change temperature, $T=0$ say, and melting is induced at time $t=0$, by raising and fixing the temperature at $x=0$ to a fixed value, e.g., $T_{0}>0$. The governing equation representing the heat conduction in the liquid domain is (see [1])

$$
\rho c \frac{\partial T}{\partial t}=-\frac{\partial q}{\partial x}, \quad 0 \leq x \leq s(t),
$$

where $q$ is the flux term, $\rho$ is density and $c$ is the specific heat (assumed constant in this work). The initial condition is $T(x>0, t=0)=0$ and the boundary conditions are $T(0, t)=T_{0}$ at $x=0$ and $T(s(t), t)=0$. Since the problem is posed in an expanding domain, an extra condition is needed at the moving interface $s(t)$

$$
q(s(t))=\rho L \frac{d s}{d t},
$$

stating that the advance of the front depends on the rate at which heat arriving at the front can supply the latent heat $L$, required to melt the solid.

Simply proposing alternative phenomenological models for the flux term $q$ will allow us to build from eqs. (1) and (2) alternative memory and non-local models. 


\section{Definitions and properties of operators}

In order to provide a high degree of flexibility in the definitions of the flux $q$

\subsection{A conventional Stefan model}

In the conventional treatment we assume that the flux can be represented by Fourier's first law

$$
q=q^{F}=-K \frac{\partial T}{\partial x},
$$


giving rise to the following governing equation and front balance condition

$$
\begin{gathered}
\rho c \frac{\partial T}{\partial t}=\frac{\partial}{\partial x}\left(K \frac{\partial T}{\partial x}\right), \quad 0 \leq x \leq s(t), \\
-\left.K \frac{\partial T}{\partial x}\right|_{x=s(t)}=\rho L \frac{d s}{d t},
\end{gathered}
$$

with

$$
\rho c \frac{\partial T}{\partial t}=\kappa \frac{\partial}{\partial x}\left(\frac{\partial}{\partial t}{ }_{0} I_{t}^{\nu, f}\left(\frac{\partial T}{\partial x}\right)\right), \quad 0 \leq x \leq s(t),
$$

where $0<\nu \leq 1$, and $\kappa$ is a scaling constant. Note on setting $f(t)=t$ we recover the flux model introduced in the memory Stefan model proposed in [7.

By using eq. (10) in our base Stefan model eqs. (1) and (2), we arrive at the following memory model

$$
-\left.\kappa \frac{\partial}{\partial t}{ }^{0} I_{t}^{\nu, f}\left(\frac{\partial T}{\partial x}\right)\right|_{x=s(t)}=\rho L \frac{d s}{d t} .
$$

Due to its conserved nature, this will be our preferred form for a memory model. Note, however, that by dividing by $f^{\prime}(t)$ and applying the fractional integral operator ${ }_{0} I_{t}{ }^{1-\nu, f}$ to both side of eqs. 11 and $\sqrt{12}$, we arrive, on using the properties of the general-Caputo definition in eqs. (4) and (5), the alternative form of memory Stefan problem

$$
\rho c_{0} \widehat{O}_{t}^{\nu, f} T=\kappa \frac{\partial^{2} T}{\partial x^{2}}, \quad 0 \leq x \leq s(t),
$$




$$
-\left.\kappa\left(\frac{\partial T}{\partial x}\right)\right|_{x=s(t)}=\rho L_{0} \widehat{O}_{t}^{\nu, f} s(t) .
$$

On setting $f(t)=t$, we recover the fractional derivative memory forms, found in the current literature (e.g., [4, 5, 6, 7, 8, 9]).

\subsection{A general non-local Stefan model}

A general non-local (GNL) model is constructed through using the general-

Caputo fractional derivative definition in eq. (4) to replace the gradient term in the Fourier law (eq. (7)), i.e.,

$$
q=q^{G N L}(x, t)=-\kappa_{0}\left({ }_{0} \widehat{O}_{x}^{\nu, f} T\right),
$$

leading to the non-local Stefan model

$$
\begin{gathered}
\rho c \frac{\partial T}{\partial t}=\kappa_{0} \frac{\partial}{\partial x}\left({ }_{0} \widehat{O}_{x}^{\nu, f} T(x, t)\right), \quad 0 \leq x \leq s(t), \\
-\kappa_{0} \widehat{O}_{x}^{\nu, f} T(s(t))=\rho L \frac{d s}{d t} .
\end{gathered}
$$

\section{A limit case Stefan model}

To arrive at analytical solutions that will expose the anomalous signals, associated with the various flux choices in the general Stefan model introduced in the previous section, we consider the limit Stefan problem, previously studied in $[4,8,9$. This is obtained by setting the density $\rho$ and latent heat $L$ to unity and letting the specific heat $c \rightarrow 0$. In the context of the one-dimensional Stefan problem studied here, the physical interpretation of the limit problem is the moisture filling (infiltration) into an initially dry, horizontal, porous tube under the application of a fixed pressure head at $x=0$. 


\subsection{The conventional limit Stefan model}

Letting $c \rightarrow 0$ in the conventional Stefan problem, eqs. (8) and (9), leads to the following limit problem

$$
\begin{gathered}
\frac{\partial}{\partial x}\left(K \frac{\partial T}{\partial x}\right)=0, \quad 0 \leq x \leq s(t), \\
-\left.K \frac{\partial T}{\partial x}\right|_{x=s(t)}=\frac{d s}{d t} .
\end{gathered}
$$

165 this problem are, $T(x>0, t=0)=0$ and boundary $T(0, t)=1, T(s(t), t)=0$ (see Fig. 1).

In a general setting, the conductivity $K$ could be a function of space or time. For example we could imagine that the media is graded such that the conductivity increases or decreases with space. Alternatively we might imagine a situation where the uniform ambient conditions are changing in such away to induce temporal changes in the conductivity. We will examine particular solutions for these two cases in detail below.

\subsection{A limit Stefan model with memory} the following limit problem

$$
\frac{\partial}{\partial x}\left(q^{F M}\right)=\frac{\partial}{\partial x}\left(\frac{\partial}{\partial t}{ }^{0} I_{t}^{\nu, f}\left(\frac{\partial T}{\partial x}\right)\right)=0, \quad 0 \leq x \leq s(t),
$$

with

$$
q^{F M}=-\left.\kappa \frac{\partial}{\partial t}{ }_{0} I_{t}{ }^{\nu, f}\left(\frac{\partial T}{\partial x}\right)\right|_{x=s(t)}=\frac{d s}{d t} .
$$

From eq. 20 we can infer, for this problem, that the memory flux $q^{F M}$, defined in eq. 10p, can only be a function of time. This in turn implies that the gradient derivative, $\partial T / \partial x$, under the time fractional integration also has to be a function of time alone. Thus, the solution for the temperature profile that satisfies the equation and boundary conditions is linear in space, i.e., 


$$
T=1-\frac{x}{s(t)}, \quad \frac{\partial T}{\partial x}=-\frac{1}{s(t)}
$$

To move forward we make the ansatz that, for any given monotonically function $f(t)$, with $f(0)=0$, the front advance is given by $s(t)=f^{\frac{\nu}{2}}$. In this

$$
q^{F M}(t)=\frac{\kappa}{\Gamma(\nu)} \frac{d}{d t} \int_{0}^{t}(f(t)-f(\tau))^{\nu-1} \frac{d}{d \tau}\left(f^{1-\frac{\nu}{2}}\right) d \tau \sim \frac{d}{d t}{ }_{0} \widehat{O}_{t}^{1-\nu, f} f^{1-\frac{\nu}{2}}
$$

Thus, by the general Caputo property in eq. (6), the memory flux in this limit case Stefan problem can be written as

$$
q^{F M} \sim \frac{d}{d t} f^{\frac{\nu}{2}}
$$

On substituting this in the front condition of eq. 21) we do indeed see that $s(t)=f^{\frac{\nu}{2}}$.

The above result imparts a high degree of utility to the Stefan memory model. Effectively, whenever the observed temperature profile is linear, this model can be used to fit any observed monotonic advance of the phase front with time. For example a setting of $f(t)=t^{m}$, with $m>0$, will fit any observed front movement with the general power-law form $s=t^{n}, n=(m \nu) / 2>0$. Further, if $f(t) \sim \ln (1+t)$, our generalized Caputo derivative in eq. (4) has the form of the regularized Hadamard derivative [18] and we can match the observation of an ultraslow motion of the melting front where $s(t) \sim \ln ^{\nu / 2}(1+t)$.

Note, in the setting of this limit problem we can, using the second component of eq. (22), rearrange the memory flux term in eq. (24):

$$
q^{F M} \sim \frac{d}{d t} f^{\frac{\nu}{2}}=f^{\prime} f^{\frac{\nu}{2}-1}=\frac{f^{\prime} f^{\nu-1}}{s}=f^{\prime}(t) f(t)^{\nu-1} \frac{\partial T}{\partial x} .
$$

From 25 we see that, this limit case Stefan problem based on the memory flux $q^{F M}$, defined in eq. 10 , is equivalent to the flux in a conventional model $q^{F}=-K \partial T / \partial x$ with a time dependent conductivity $K(t)=f^{\prime}(t) f(t)^{\nu-1}$. 


\subsection{A limit non-local Stefan model}

Letting $c \rightarrow 0$ in the non-local Stefan problem, eqs. (16) and (17), leads to the following limit problem

$$
\begin{gathered}
\frac{\partial}{\partial x}\left(q^{G N L}\right)=\frac{\partial}{\partial x}\left({ }_{0} \widehat{O}_{x}^{\nu, f} T(x, t)\right)=0, \quad 0 \leq x \leq s(t), \\
q^{G N L}=-\kappa_{0} \widehat{O}_{x}^{\nu, f} T(s(t))=\frac{d s}{d t} .
\end{gathered}
$$

Now we see that the non-local flux $q^{G N L}$ can only be a function of time $t$. By the property eq. (5) of the general Caputo derivative, eq. (4), this requires, on accounting for the boundary conditions, that

$$
\left.T(x, t)=1-\frac{f(x)^{\nu}}{f(s)^{\nu}}, \quad{ }_{0} \widehat{O}_{x}^{\nu, f} T(x, t)\right) \sim \frac{1}{f(s)^{\nu}} .
$$

In this way, if we restrict ourselves to power-law functions with the form $f(x)=$ $x^{m}$, with $m>0$, we can readily generate power-law in time predictions for the advance of the phase front as $s=t^{n}, 0 \leq n=1 /(1+m \nu) \leq 1$.

There are two point to make here. First, in contrast to the general-memory approach, the ability of the general non-local approach, to match a given monotonic advance of the phase front is restricted to power-laws in time, with exponents in the range $0<n \leq 1$. Secondly, again in contrast to the general-memory approach, the general non-local approach will always generate a curved temperature profile, eq. (28). A linear profile is only recovered under normal-diffusion conditions, $\nu=1, f(x)=x$.

Note further, in considering the limit case Stefan problem with $f(x)=x^{m}$, ${ }_{220} m>0$, if we set a non linear conductivity as $K(x)=f^{(1-\nu)} / f^{\prime}=x^{1-m \nu}$ we see that our non-local flux is equivalent to a local flux with a non-linear conductivity, i.e., within an appropriate constant, $q^{G N L} \equiv K(x)(\partial T / \partial x)$.

\section{Discussion}

In this work our focus has been to study the influence of the flux definition 

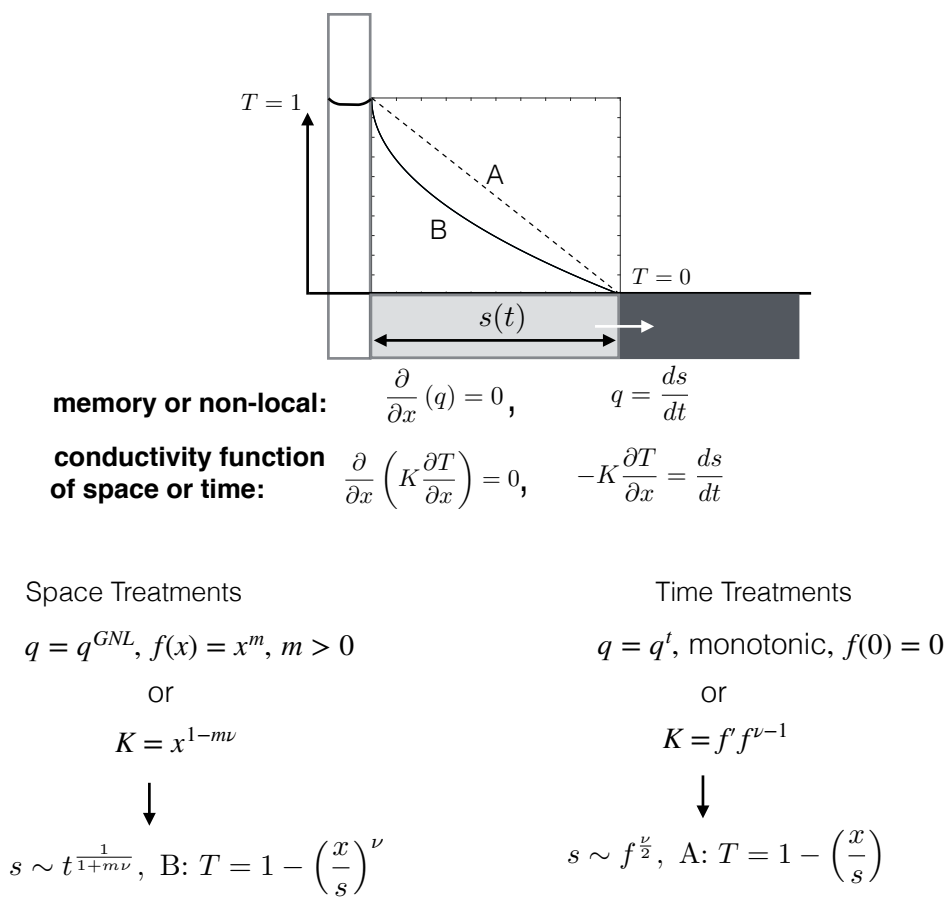

Figure 1: Model choices for limit case Stefan problem

$s(t)$ from a one-dimensional one-phase Stefan melting problem. We select three different flux definitions to investigate; (i) a conventional Fourier law (conductivity $\times$ temperature gradient) with a conductivity as function of time (aging) or space (graded material), (ii) a flux that can account for system memory expressed in terms of an order $0<\nu \leq 1$ fractional derivative of the temperature with respect to a general function of time $f(t)$, and (iii) a flux that can account for non-locality, expressed in terms of an order $0<\nu \leq 1$ fractional derivative of the temperature with respect to a general function of space $f(x)$. All of these flux definitions can produce anomalous diffusion signals in which the monotonic advance of the melting front differs from the normally expected value of $1 / 2$.

As we have noted in our introduction, recent work on Stefan problems [4] has suggested that, in matching a given anomalous observation, there is some ambiguity in selecting an appropriate flux definition. For example, an observed anomalous front advance can be independently matched by two of the three 


\section{Acknowledgments}

This research is supported by the Basque Government through the BERC 2014-2017 and BERC 2018-2021 programs, and by the Spanish Ministry of Economy and Competitiveness MINECO through BCAM Severo Ochoa excellence accreditations SEV-2013-0323 and SEV-2017-0718. The research began and was primarily developed at BCAM, Bilbao, during the visiting fellowship of Roberto Garra in August-October 2017. 


\section{References}

[1] J. Crank, Free and moving boundary problems, Oxford University Press, 1987.

[2] Y.-X. Tao, R. W. Besant, K. S. Rezkallah, A mathematical model for predicting the densification and growth of frost on a flat plate, Int. J. Heat Mass Transfer 36 (1993) 353-363.

[3] N. Filipovitch, K. M. Hill, A. Longjas, V. R. Voller, A mathematical model for predicting the densification and growth of frost on a flat plate, Water Resources Research 52 (2016) 5167-5178.

[4] F. Falcini, R. Garra, V. R. Voller, Modeling anomalous heat diffusion: Comparing fractional derivative and non-linear diffusivity treatments, International Journal of Thermal Sciences 137 (2019) 584-588.

[5] A. N. Ceretani, D. A. Tarzia, Determination of two unknown thermal coefficients through an inverse one-phase fractional stefan problem, Fractional Calculus and Applied Analysis 20 (2017) 399-421.

[6] S. Roscani, E. Marcus, Two equivalent stefan's problems for the time fractional diffusion equation, Fractional Calculus and Applied Analysis 16 (2013) 802-815.

[7] V. R. Voller, F. Falcini, R. Garra, Fractional stefan problems exhibiting lumped and distributed latent-heat memory effects, Physical Review E 87 (2013) 042401.

[8] V. R. Voller, An exact solution of a limit case stefan problem governed by a fractional diffusion equation, International Journal of Heat and Mass Transfer 53 (2010) 5622-5625.

[9] V. R. Voller, Fractional stefan problems, International Journal of Heat and Mass Transfer 74 (2014) 269-277. 
[10] V. R. Voller, F. Falcini, Fractional stefan problems, International Journal of Heat and Mass Transfer 58 (2013) 80-85.

[11] A. Ceretani, A note on models for anomalous phase-change processes, arXiv preprint arXiv:1801.10069.

[12] V. R. Voller, Anomalous heat transfer: Examples, fundamentals, and fractional calculus models, Advances in Heat Transfer 50 (2018) 333-380.

[13] A. A. Kilbas, H. M. Srivastava, J. J. Trujillo, Theory and Applications of Fractional Differential Equations, Vol. 204, North-Holland Mathematics Studies, 2006.

[14] A. S. Bodrova, A. V. Chechkin, A. G. Cherstvy, R. Metzler, Ultraslow scaled brownian motion, New Journal of Physics 17 (2015) 063038.

[15] G. Pagnini, Erdélyi-kober fractional diffusion, Fractional Calculus and Applied Analysis 15 (2012) 117-127.

[16] D. Molina-García, T. M. Pham, P. Paradisi, C. Manzo, G. Pagnini, Fractional kinetics emerging from ergodicity breaking in random media, Physical Review E 94 (2016) 052147.

[17] R. Almeida, A caputo fractional derivative of a function with respect to another function, Communications in Nonlinear Science and Numerical Simulation 44 (2017) 460-481.

[18] F. Jarad, T. Abdeljawad, D. Baleanu, A caputo fractional derivative of a function with respect to another function, Advances in Difference Equations $2012(2012) 142$. 